\title{
Correction: Lim et al., Optogenetic Mapping after Stroke Reveals Network-Wide Scaling of Functional Connections and Heterogeneous Recovery of the Peri-Infarct
}

In the article "Optogenetic Mapping after Stroke Reveals Network-Wide Scaling of Functional Connections and Heterogeneous Recovery of the Peri-Infarct” by Diana H. Lim, Jeffrey M. LeDue, Majid H. Mohajerani, and Timothy H. Murphy, which appeared on pages 16455-16466 of the December 3, 2014 issue, the authors noticed an error in Figure 7A. In the process of manuscript preparation, the 1 week stroke recovery plot for Figure $7 A$ was inadvertently switched with a plot of 8 week stroke recovery data. Corrected Figure 7 is provided below. All original conclusions and statistics reported in the text were based on 1 and 8 week stroke recovery data respectively, and are not affected by the error in figure presentation.
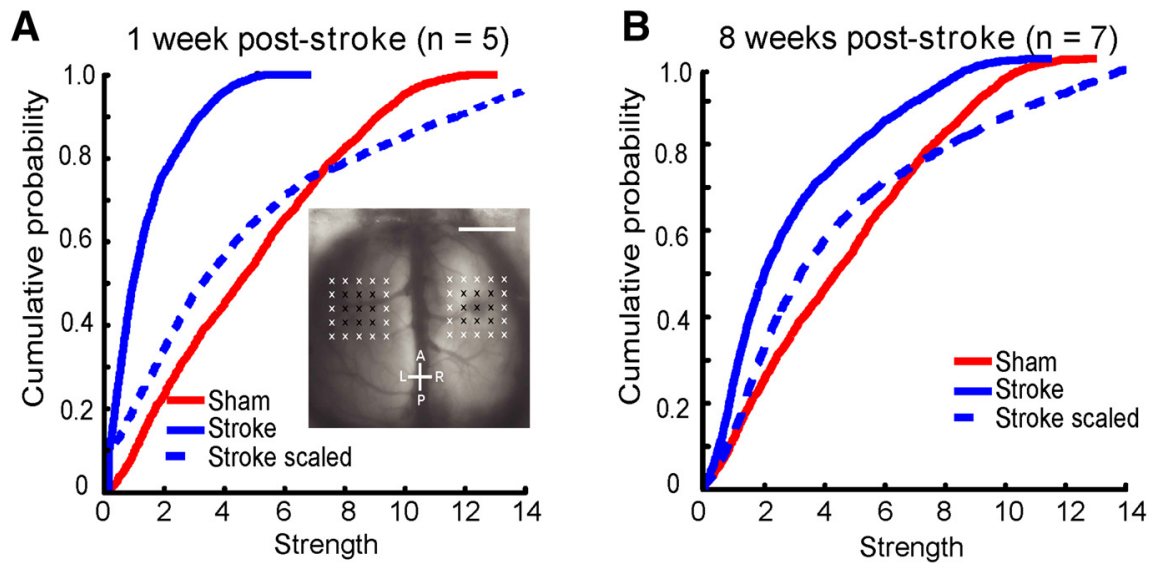

Figure 7.

DOI:10.1523/JNEUROSCI.1856-15.2015 